\title{
Short communication: Herd-level prevalence of Mycobacterium avium ssp. paratuberculosis is not associated with participation in a voluntary Alberta Johne's disease control program
}

\author{
C. Ritter, ${ }^{* 1}$ R. Wolf, ${ }^{*}$ C. L. Adams, † D. F. Kelton, $\ddagger$ C. Pickel, ${ }^{\star}$ S. Mason, ${ }^{*} \S$ K. Orsel, ${ }^{*}$ J. De Buck, ${ }^{*}$ \\ and H. W. Barkema* \\ *Department of Production Animal Health, and \\ †Department of Veterinary Clinical and Diagnostic Sciences, Faculty of Veterinary Medicine, University of Calgary, Calgary, AB, Canada, T2N 4N1 \\ ‡Department of Population Medicine, Ontario Veterinary College, University of Guelph, Guelph, ON, Canada, N1G 2W1 \\ $\S$ AgroMedia International Inc., Calgary, AB, Canada, T2L 0T6
}

\section{ABSTRACT}

Johne's disease (JD) control programs for dairy farms have the general objective of reducing both cowand herd-level prevalence of Mycobacterium avium ssp. paratuberculosis (MAP). An important aspect of many programs is herd testing for MAP to determine the infection status of participating farms. However, it is uncertain whether MAP herd-level prevalence on farms voluntarily participating in a JD control program is different from that on nonparticipating farms. Therefore, the aim was to compare MAP infection status of participants and nonparticipants in the Alberta Johne's Disease Initiative (AJDI), a voluntary JD control program initiated in 2010 in Alberta, Canada. Between September 2012 and August 2013, environmental fecal samples were collected from 93 randomly selected farms not enrolled in the AJDI. Additionally, 81 farms that initially enrolled in the AJDI during the same time interval were also sampled. Samples were collected from 6 defined locations on each farm and cultured for MAP. Results were confirmed using conventional IS900 PCR and F57 quantitative PCR. Overall, $51 \%$ of participating and $51 \%$ of nonparticipating farms were identified as being MAP-infected. Furthermore, based on multivariable logistic regression, the number of MAP-positive samples was not associated with AJDI participation (taking herd size into account as a potentially modifying or confounding variable). In conclusion, there was no indication that voluntary participation in the AJDI was associated with herd-level MAP prevalence.

Key words: prevalence, Mycobacterium avium ssp. paratuberculosis, Alberta Johne's Disease Initiative, behavior

Received September 6, 2015.

Accepted November 21, 2015

${ }^{1}$ Corresponding author: cmnritte@ucalgary.ca

\section{Short Communication}

Johne's disease (JD) is an infectious, chronic enteritis that can affect most ruminants (McKenna et al., 2006), and it is caused by Mycobacterium avium ssp. paratuberculosis (MAP). Clinical signs include reduced milk production, progressive diarrhea, and weight loss, resulting in financial losses for the producer (Wolf et al., 2014b). Additionally, MAP can be present in milk for human consumption (Okura et al., 2012), and there is a potential association of MAP with Crohn's disease in humans (Barkema et al., 2011). Currently, prevention and control programs focus on decreasing transmission of MAP within a farm and between farms (Sweeney et al., 2012; Barkema et al., 2014). Therefore, many Canadian provinces have implemented voluntary management-based control programs for JD. The Alberta Johne's Disease Initiative (AJDI) was launched in 2010, with approximately $65 \%$ of Alberta's 580 dairy farms participating by 2013 (Wolf et al., 2014a). In addition to comprehensive extension efforts from the industry, dairy practitioners in Alberta were encouraged to motivate farmers' enrollment. Furthermore, practitioners had the option to become AJDI-certified and conduct annual risk assessments to identify and reduce high-risk management practices for MAP transmission on producers' farms (Wolf et al., 2014a). Environmental fecal samples were collected (concurrent with risk assessments) and cultured for MAP.

A recent study detected MAP on $47 \%$ of AJDI participating farms; however, the true prevalence was estimated to be $69 \%$ (Wolf et al., 2014a). It is unclear whether this estimate is representative of all Alberta dairy farms, or if there are distinct differences in MAP herd-level prevalence between farms participating in AJDI versus nonparticipating farms. These 2 distinct farmer groups differed in some of their attitudes toward JD and the AJDI (Ritter et al., 2015), and attitudes have been associated with the incidence of other cattle 
diseases (Jansen et al., 2009). In a similar voluntary JD control program in Ontario, the herd-level prevalence of MAP was approximately $5 \%$ higher in nonparticipating herds, based on bulk tank milk ELISA testing (Kelton et al., 2014). If MAP was indeed less present on AJDI participating farms, the true prevalence in Alberta dairy herds would be even higher than estimated by Wolf et al. (2014a). This finding would have important implications for extension strategies. Whereas every participating farm is an asset to JD control programs, to reduce the risk of MAP transmission and enhance success of the program, there should be increased efforts to encourage more hesitant farmers to enroll. Therefore, the objective of this study was to compare MAP herd-level infection status between farms enrolled and not enrolled in the AJDI.

Between September 2012 and August 2013, 122 farmers not enrolled in the AJDI (i.e., nonparticipants) were randomly selected (the number of nonparticipating farms decreased from 292 to 206 farms over the course of the study). Selected nonparticipants were contacted by telephone and asked to take part in the study. Ninety-three $(76 \%)$ of the contacted nonparticipants agreed to environmental sampling on their farm and to provide demographic information. During the same time window, all 81 AJDI participating dairy herds that had their initial risk assessment and environmental sampling on their farm were included in the study. Sampling during the same timeframe mitigated bias due to ongoing improvements in laboratory procedures for identifying MAP (Wolf et al., 2014a) and due to changes in farmers' risk-associated behavior as a response to ongoing province-wide JD education.

Sample collection on nonparticipating farms was done by trained University of Calgary and Alberta Milk (Edmonton, AB, Canada) personnel, whereas participating farms were sampled by herd veterinarians. All personnel and veterinarians collecting samples and conducting interviews underwent AJDI-specific training. Detailed descriptions of sample collection and laboratory analyses have been reported (Wolf et al., 2014a). In short, duplicate environmental fecal samples were collected from 3 areas: (1) manure concentration (e.g., alleys); (2) cow concentration (e.g., sick pen); and (3) manure storage (e.g., lagoons, piles, or pits). If there were fewer than 2 cows in a pen or if manure could not be collected from manure storage, additional samples from remaining areas were collected to obtain 6 samples from each farm. Samples were processed using a standardized 3-d decontamination protocol, followed by $48 \mathrm{~d}$ of culture using a Trek ESP culture protocol (Trek Diagnostic Systems Inc., Independence, OH; Mortier et al., 2014). Conventional insertion sequence 900 (IS900) PCR was used to confirm culture results. If IS900 results were inconclusive, sequence element F57 quantitative PCR (qPCR) was applied for clarification. Therefore, the case definition was positive on either IS900 conventional PCR or F57 qPCR.

Statistical analyses were done with Stata SE 12 (StataCorp LP, College Station, TX) and $P<0.05$ was considered significant. First, descriptive statistical analyses were performed. Numbers of MAP-negative and MAP-positive farms (i.e., zero versus at least one environmental fecal sample was MAP-positive) were compared, and differences between AJDI participants and nonparticipants were assessed using a chi-squared test. Then, numbers of MAP-positive samples on participating and nonparticipating farms were compared using the Mann-Whitney U-test. This comparison was done using all 6 samples per farm and by grouping farms into low, moderate, or high MAP infection status ( 1 or 2,3 or 4 , and 5 or 6 of the 6 samples positive, respectively).

Herd size, represented by the number of lactating cows, was evaluated as a potential confounder and effect modifier of the relationship between number of MAPpositive samples and AJDI participation. In previous studies, larger herds were more likely to be infected with MAP compared with smaller herds (USDA, 2007; Pillars et al., 2009; Wolf et al., 2014a). Also, herds participating in 2 Canadian JD control programs were larger than nonparticipating herds (Kelton et al., 2012; Ritter et al., 2015). Information on number of lactating cows was self-reported and obtained through a face-toface interview completed concurrent with environmental sample collection or during AJDI risk assessment for nonparticipants and participants, respectively. Univariable ordinal logistic regression analysis was done to determine if herd size was predictive of number of MAP-positive samples. Herd size was also assessed in univariable logistic regression analysis for association with AJDI participation among MAP-negative farms.

Finally, a multivariable logistic regression model was developed to assess the relationship between AJDI participation (i.e., outcome) and number of MAP-positive samples (i.e., exposure), taking into account herd size and the interaction of herd size and exposure. Manual backward elimination of putative explanatory variables was done to arrive at the final model. "Herd size" was considered a confounder if the coefficient of exposure (i.e., number of MAP-positive samples) changed by $>30 \%$.

Overall, 47 (51\%) nonparticipating and 41 (51\%) participating farms were designated MAP-infected $(P$ $=0.99)$. Specifically, $16 \%$ of nonparticipating farms and $15 \%$ of participating farms had 1 MAP-positive en- 
vironmental sample, whereas 9 and $14 \%$, respectively, had the maximum of 6 positive samples (Figure 1). The number of MAP-positive samples out of 6 environmental samples was not different between AJDI participants and nonparticipants $(P=0.45)$. Similarly, proportions of farms with a low, moderate, or high MAP infection status was not different between groups $(P=0.50)$.

Farms that enrolled in the AJDI between September 2012 and August 2013 had a median of 95 lactating cows $($ mean $=110, \mathrm{SD}=64)$, whereas nonparticipants had a median of 90 lactating cows (mean $=104, \mathrm{SD}$ $=70$; Figure 2). Based on regression analyses, herd size was not predictive of participation among MAPnegative farms $(P=0.61 ; 95 \% \mathrm{CI}=-0.73$ to 1.25$)$, but was positively associated with number of positive samples $(P<0.001 ; 95 \% \mathrm{CI}=0.50$ to 1.60$)$. Whereas the latter result confirmed previous reports (USDA, 2007; Wolf et al., 2014a), it was surprising that herd size was not associated with participation in the AJDI. An explanation for this discrepancy is that the AJDI was launched in 2010, but only farmers that enrolled between September 2012 and August 2013 were eligible for this study. Farms enrolled before September 2012 had a median of 99 lactating cows (mean $=117, \mathrm{SD}$ $=68)$. For these farms, herd size was positively associated with participation among MAP-negative herds $(P$ $=0.02$ ). In contrast, the current study included farms more similar in herd size to nonparticipating farms. The larger herd size of farms enrolling early on in the AJDI and the association between herd size and MAP infection status argues for a higher herd-level prevalence of MAP on early-participating farms that were not included in the study. Unfortunately, continuous improvement of test sensitivity over the course of the AJDI precluded valid comparison of MAP presence in samples collected at various time points. However, $51 \%$ of the 214 participating farms that already had their second or third round of testing done between September 2012 and August 2013 were MAP-positive. This percentage was identical to the proportion of positive farms that had their first testing done during this time window, and therefore does not provide proof for the assumption that early participants had higher MAP infection status. Nonetheless, perhaps this group of farmers already implemented management strategies for MAP reduction, as recommended during risk assessments. In particular, culling affected cows could have reduced the within-herd MAP prevalence.

In the multivariable regression analysis, an increase in herd size did not affect the association between number of MAP-positive samples and participation status $(P=$ 0.57). However, removing "herd size" from the model changed the exposure coefficient by $45 \%$. Therefore, herd size was considered a confounder for the relation-

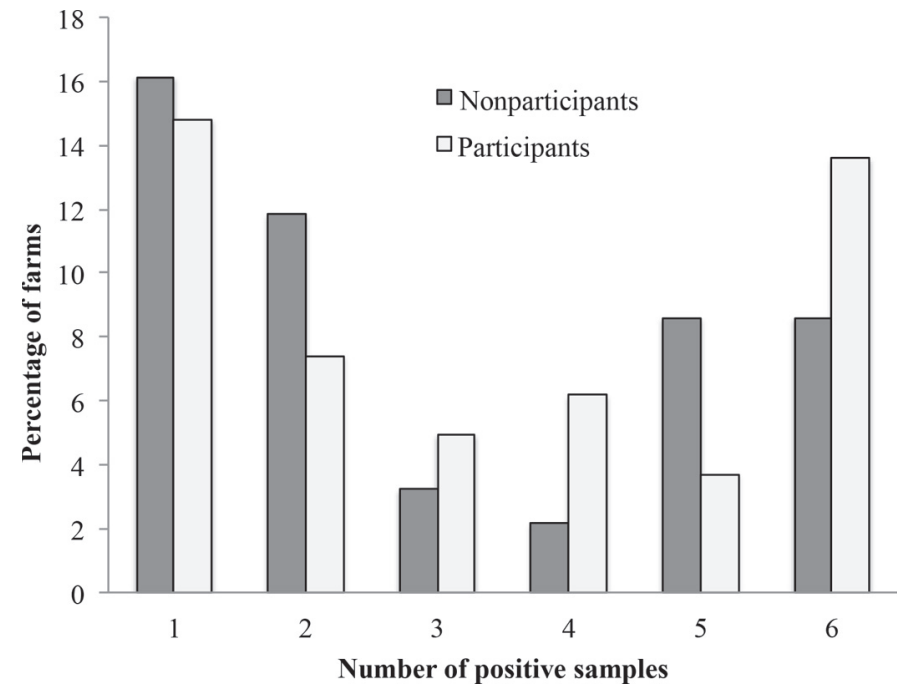

Figure 1. Percentage of Mycobacterium avium ssp. paratuberculosis-positive environmental samples on 81 farms participating and 93 farms not participating in the Alberta Johne's Disease Initiative.

ship between MAP-positive samples and participation status, and it was forced into the final multivariable regression model. Thus, for every additional MAPpositive sample, there was a $1.02(P=0.79 ; 95 \% \mathrm{CI}=$ 0.88 to 1.18 ) increase in odds of participation.

In this study, MAP was identified on $51 \%$ of AJDI participating farms, very similar to the apparent prevalence of $47 \%$ recently reported (Wolf et al., 2014a).

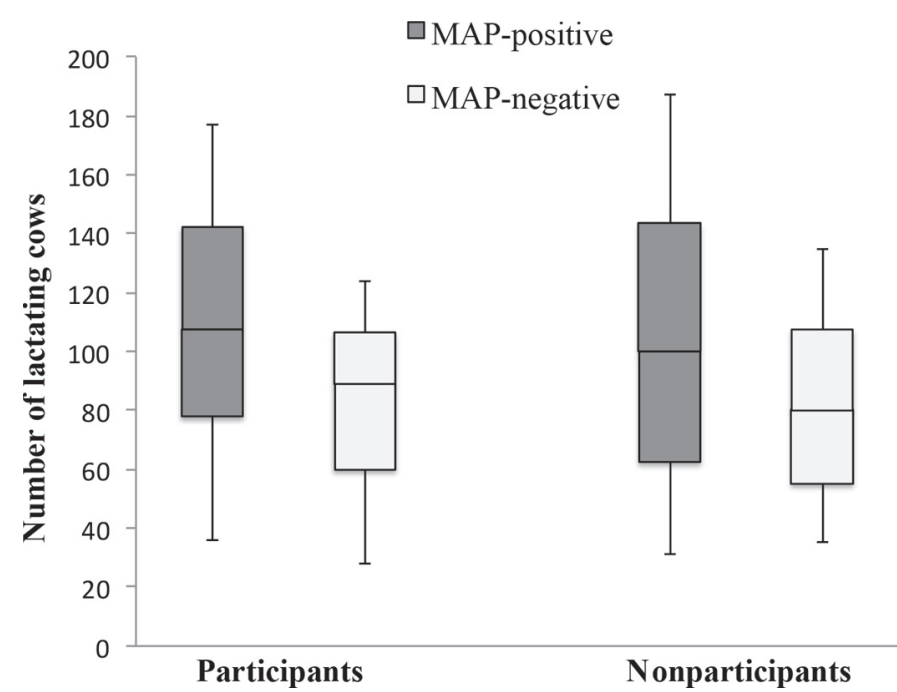

Figure 2. Number of adult dairy cows in herds that were Mycobacterium avium ssp. paratuberculosis (MAP)-positive or MAPnegative on farms participating or not participating in the Alberta Johne's Disease Initiative. The bottom and top of the box are the first and third quartile, respectively. The band inside the box represents the median. The ends of the whiskers mark the lowest and highest value within $1.5 \times$ interquartile range of the first and third quartile, respectively. 
Furthermore, the numbers of MAP-positive samples on AJDI participating and nonparticipating farms were comparable. Therefore, we concluded that the AJDI was adopted by farms with a MAP infection status that was similar to that of the general dairy population.

Participants in a similar JD control program in Ontario were $5 \%$ less often positive on the bulk tank milk ELISA test (Kelton et al., 2014). Furthermore, these farmers had better herd performance compared with nonparticipants (e.g., higher milk production and lower bulk tank SCC; Kelton et al., 2014). In contrast, a comparison of participants' and nonparticipants' management characteristics in Alberta did not detect these differences (Ritter et al., 2015). Although JD programs in these 2 Canadian provinces are based on the same principles, there are slight differences in implementation. For example, environmental manure sampling is done in Alberta, whereas individual cow testing (ELISA on milk or serum) is used in Ontario. Also, farmers in Ontario only receive reimbursement for testing if they permanently remove high-titer cows (Kelton et al., 2012). These differences in program composition could have affected participation and contributed to the apparent divergence in results.

In conclusion, the concern that voluntary JD control programs attract mostly farms with better MAP infection status was not confirmed for Alberta dairies, as neither MAP herd-level prevalence nor number of positive environmental fecal samples per farm was associated with participation in the AJDI.

\section{ACKNOWLEDGMENTS}

The authors thank the dairy producer organization Alberta Milk (Edmonton, AB, Canada) for their assistance with farm recruitment and sample collection. This study was funded by Alberta Milk (Edmonton, AB, Canada), the Alberta Livestock and Meat Agency Ltd. (Edmonton, AB, Canada), and Canada's Natural Sciences and Engineering Research Council (NSERC) Industrial Research Chair Program, with industry contributions from Alberta Milk, the Dairy Farmers of Canada (Ottawa, ON, Canada), Dairy Farmers of Manitoba (Winnipeg, MN, Canada), British Columbia Dairy Association (Burnaby, BC Canada), Westgen Endowment Fund (Milner, BC, Canada), Canadian Dairy Network (Guelph, ON, Canada), and CanWest DHI (Guelph, ON, Canada).

\section{REFERENCES}

Barkema, H. W., S. Hendrick, J. M. De Buck, S. Ghosh, G. G. Kaplan, and K. P. Rioux. 2011. Crohn's disease in humans and Johne's disease in cattle - Linked diseases? Pages 197-213 in Zoonotic Pathogens in the Food Chain. D. Krause and S. Hendrick, ed. CAB International, Wallingford, UK.

Barkema, H. W., K. Orsel, A. Koets, V. P. Rutten, J. Bannantine, G. P. Keefe, D. F. Kelton, S. J. Wells, R. Whittington, C. G. Mackintosh, E. J. Manning, M. F. Weber, J. P. Kastelic, and J. De Buck. 2014. Bottlenecks in the prevention and control of Mycobacterium avium ssp. paratuberculosis infection. Pages 111-113 in Proc. 12th Int. Colloq. Paratuberculosis, Parma, Italy.

Jansen, J., B. H. P. Van den Borne, R. J. Renes, G. Van Schaik, T. J. G. M. Lam, and C. Leeuwis. 2009. Explaining mastitis incidence in Dutch dairy farming: The influence of farmers' attitudes and behaviour. Prev. Vet. Med. 92:210-223.

Kelton, D., N. Perkins, A. Godkin, G. MacNaughton, R. Cantin, and K. Hand. 2012. Comparison of participants and non-participants in a voluntary Johne's disease control program in Ontario, Canada. Pages 151-154 in Proc. 11th Int. Colloq. Paratuberculosis, Sydney, Australia.

Kelton, D., N. Perkins, A. Godkin, G. MacNaughton, R. Cantin, K. Hand, and M. Watters. 2014. Comparison of participants and non-participants in a voluntary Johne's disease control program in Ontario, Canada. Page 142 in Proc. 12th Int. Colloq. Paratuberculosis, Parma, Italy.

McKenna, S. L., G. P. Keefe, A. Tiwari, J. VanLeeuwen, and H. W. Barkema. 2006. Johne's disease in Canada part II: Disease impacts, risk factors, and control programs for dairy producers. Can. Vet. J. 47:1089-1099.

Mortier, R. A., H. W. Barkema, K. Orsel, R. Wolf, and J. De Buck. 2014. Shedding patterns of dairy calves experimentally infected with Mycobacterium avium subspecies paratuberculosis. Vet. Res. 45:71.

Okura, H., N. Toft, and S. S. Nielsen. 2012. Occurrence of Mycobacterium avium ssp. paratuberculosis in milk at dairy cattle farms: A systematic review and meta-analysis. Vet. Microbiol. 157:253-263.

Pillars, R. B., D. L. Grooms, J. A. Woltanski, and E. Blair. 2009. Prevalence of Michigan dairy herds infected with Mycobacterium avium subspecies paratuberculosis as determined by environmental sampling. Prev. Vet. Med. 89:191-196.

Ritter, C., G. P. S. Kwong, R. Wolf, C. Pickel, M. Slomp, J. Flaig, S. Mason, C. L. Adams, D. F. Kelton, J. Jansen, J. De Buck, and H. W. Barkema. 2015. Factors associated with participation of Alberta dairy farmers in a voluntary, management-based Johne's disease control program. J. Dairy Sci. 98:7831-7845.

Sweeney, R. W., M. T. Collins, A. P. Koets, S. M. McGuirk, and A. J. Roussel. 2012. Paratuberculosis (Johne's disease) in cattle and other susceptible species. J. Vet. Intern. Med. 26:1239-1250.

USDA. 2007. Johne's disease on U.S. dairies, 1991-2007. USDAAPHIS-VS-CEAH-NAHMS. Accessed Oct. 12, 2015. https://www.aphis.usda.gov/animal_health/nahms/dairy/ downloads/dairy07/Dairy07_is_Johnes.pdf.

Wolf, R., H. W. Barkema, J. De Buck, M. Slomp, J. Flaig, D. Haupstein, C. Pickel, and K. Orsel. 2014a. High herd-level prevalence of Mycobacterium avium subspecies paratuberculosis in Western Canadian dairy farms, based on environmental sampling. J. Dairy Sci. 97:6250-6259.

Wolf, R., F. Clement, H. W. Barkema, and K. Orsel. 2014b. Economic evaluation of participation in a voluntary Johne's disease prevention and control program from a farmer's perspective-The Alberta Johne's Disease Initiative. J. Dairy Sci. 97:2822-2834. 